\title{
Cerebrospinal Fluid Biomarkers in Familial Forms of Alzheimer's Disease and Frontotemporal Dementia
}

\author{
Nina Rostgaard Gunhild Waldemar Jørgen Erik Nielsen \\ Anja Hviid Simonsen \\ Danish Dementia Research Centre, Department of Neurology, Section 6911, Rigshospitalet, \\ Copenhagen University Hospital, Copenhagen, Denmark
}

\section{Key Words}

Biomarkers · Cerebrospinal fluid · Alzheimer's disease · Frontotemporal dementia · Tau protein · Amyloid- $\beta$ - Diagnosis - Familial dementia

\begin{abstract}
As dementia is a fast-growing health care problem, it is becoming an increasingly urgent need to provide an early diagnosis in order to offer patients the best medical treatment and care. Validated biomarkers which reflect the pathology and disease progression are essential for diagnosis and are important when developing new therapies. Today, the core protein biomarkers amyloid- $\beta_{42}$, total tau and phosphorylated tau in the cerebrospinal fluid (CSF) are used to diagnose Alzheimer's disease (AD), because these biomarkers have shown to reflect the underlying amyloid and tau pathology. However, the biomarkers have proved insufficient predictors of dementias with a different pathology, e.g. frontotemporal dementia (FTD); furthermore, the biomarkers are not useful for early AD diagnosis. Familial dementias with a known disease-causing mutation can be extremely valuable to study; yet the biomarker profiles in patients with familial dementias are not clear. This review summarizes CSF biomarker findings from studies on symptomatic and presymptomatic individuals carrying a mutation in one of the genes known to cause early-onset familial AD or FTD. In conclusion, the biomarker profile of inherited $A D$ is quite similar between carriers of different mutations as well as similar to the profile found in sporadic AD, whereas familial FTD does not seem to have a clear biomarker profile. Hence, new biomarkers are needed for FTD.
\end{abstract}

(C) 2015 S. Karger AG, Basel 


\section{Introduction}

Alzheimer's disease (AD) and frontotemporal dementia (FTD) are neurodegenerative disorders characterized by decline in memory and other cognitive abilities. Nearly 40 million people worldwide are affected by dementia, and this number is estimated to double every 20 years, thus giving rise to a growing global socioeconomic and medical problem [1]. AD is the most common form of dementia in elderly people ( $>65$ years) and arises sporadically in most cases. Familial dementias are rare, and autosomal dominantly inherited AD only comprises $1-3 \%$ of all AD cases, whereas between $20-40 \%$ of FTD cases are familial [2, 3].

Familial AD is caused by autosomal dominant mutations in one of the three identified risk genes amyloid precursor protein (APP), presenilin 1 (PSEN1) and PSEN2. These mutations are autosomal dominantly inherited, with an age-dependent complete penetrance resulting in alterations of amyloid- $\beta(A \beta)$ processing and development of early-onset AD (EOAD) with clinical symptoms before 65 years of age.

The clinical presentation of FTD differs from that of AD in various behavioural symptoms and language functional deficits, which are the most common symptoms [4]. Mutations in $M A P T, G R N, T A R D B P, V C P, F U S$ and $C H M P 2 B$ and a recently identified C9ORF72 hexanucleotide repeat expansion all cause early-onset familial FTD with similar clinical presentations, whereas the underlying protein pathology, although leading to neurodegeneration, is different between the genetic subtypes.

In typical cases, the clinical diagnosis of AD, FTD and other types of dementia is based on medical history, physical and neurological examination, structural neuroimaging, neuropsychological tests and blood biochemistry. The core cerebrospinal fluid (CSF) biomarkers used for $\mathrm{AD}$ diagnosis $\left[\mathrm{A} \beta_{42}\right.$, total tau ( $\mathrm{t}$-tau) and phosphorylated tau (p-tau)] may be applied in parallel with imaging markers using MRI, fluorodeoxyglucose or amyloid PET scans for early and more accurate diagnosis $[5,6]$. In sporadic AD, the CSF profile is normally characterized by decreased levels of $A \beta_{42}$, reflecting the aggregation of $A \beta$ into insoluble plaques, and increased levels of both t-tau and p-tau as a result of neuronal damage. A large subset of FTD cases has pathological tau changes, with robust increases in CSF t-tau and p-tau levels but normal levels of $A \beta_{42}$ [4]. However, the biomarkers lack specificity, and a vast clinical overlap between normal aging, AD, FTD and other neurodegenerative diseases makes it difficult to distinguish these diseases and other forms of dementia disorders from one another. Patients may be misdiagnosed and consequently left without access to appropriate treatment and care; therefore, a new battery of biomarkers is highly needed.

A majority of biomarker discovery and validation studies have been performed on CSF from patients with sporadic AD and FTD, and although familial forms of dementia are rare in the population compared to the sporadic forms, knowledge of biomarkers within these disorders may help elucidate the disease pathology of the sporadic diseases. Furthermore, familial forms of dementia can be studied in a presymptomatic state, which affords a unique opportunity to assess biomarker profiles before symptom onset. Biomarker profiles reflecting early disease pathogenesis would be valuable in the attempt to provide an early diagnosis for individuals presenting to the clinic with mild cognitive problems. This systematic review summarizes published reports on CSF biomarker findings in patients with familial AD and FTD.

\section{Methods}

Search Strategy and Selection Criteria

A PubMed and Google Scholar search up to September 2014 was performed, using the search term 'CSF AND biomarker AND xx mutation carriers', $\mathrm{xx}$ being one of the following genes: PS1/PSEN1, PS2/PSEN2, APP, 
MAPT, GRN, TARDBP, VCP, CHMP2B, FUS and C9ORF72; subsequently, the results were reviewed for relevance. Only publications in the English language were evaluated. Articles were selected if (a) they were on human subjects, (b) they contained biomarker data on CSF and (c) individuals with mutations in one of the above-mentioned genes were included in the study, both symptomatic and presymptomatic cases. Case studies were included.

\section{Results}

In total, 44 research papers were of relevance for this review (online suppl. table 1; for all online suppl. material, see www.karger.com/doi/10.1159/000381828); 27 described familial AD and 17 described familial FTD. Several studies included more than one gene/ mutation. Only papers on patients with disease-causing mutations in the genes, with described changes in CSF biomarkers, were reviewed.

Eighteen of the 40 papers were case studies with 1 or 2 mutation carriers included. In 6 studies, 20 or more mutation carriers were included. In the majority of studies, immunebased assays were used to assess biomarker levels. In 3 studies, mass spectrometry was used.

\section{Biomarker Profiles in Familial AD}

In both symptomatic and presymptomatic APP, PSEN1 and PSEN2 mutation carriers, the majority of the studies found a decrease in $A \beta_{42}$ and an increase in both t-tau and p-tau levels when compared with healthy control individuals, consistent with a typical AD profile [7-26] (table 1). In some cases, 1 or more markers were found to be unchanged compared to controls [27-30]. In 1 study, symptomatic carriers of PSEN1 mutations had significantly lower mean levels of CSF $A \beta_{42}$ compared to patients with sporadic EOAD, sporadic late-onset AD and healthy controls, but no difference in respect to both t-tau and p-tau levels was found between the symptomatic mutation carriers and the patients with the sporadic forms of the disease. Interestingly, levels of grey matter loss as assessed by structural MRI were equal between mutation carriers and sporadic EOAD patients, thus revealing a lack of correlation between total brain amyloid load and the degree of cerebral atrophy [19]. In addition to the standard CSF biomarkers, a few studies have analysed other proteins as potential biomarkers. In individuals carrying APP or PSEN1 mutations, levels of F2-isoprostanes, which are markers of lipid peroxidation, were found to be elevated compared with controls [20]. In another study, a marker of neuronal death, VILIP-1, was found to be increased in carriers of APP, PSEN1 and PSEN2 mutations [13]. In 3 studies, mass spectrometry was used to assess $A \beta$ isoforms and the proteome in familial AD $[9,31,32]$.

\section{Biomarker Profiles in Familial FTD}

Individuals with MAPT G272V, P301L and R406W mutations were found to have normal levels of $A \beta_{42}$, and in one study elevated levels of tau and p-tau [33-35] (table 1). Some individuals with familial FTD caused by GRN mutations had a biomarker profile typical of AD, with decreased $A \beta_{42}$ and increased p-tau and t-tau CSF levels [36, 37]. However, in other cases, $\mathrm{p}$-tau and t-tau levels were within normal range $[38,39]$. C9ORF72 repeat expansion carriers were found to have either CSF profiles within a normal range or profiles similar to those of $A D$ patients, with decreased $A \beta_{42}$ and increased p-tau and t-tau levels [39-43]. Another biomarker candidate, phosphorylated TDP-43, was measured in patients with GRN or C9ORF72 mutations and was found to be higher in these individuals when compared to sporadic FTD patients [39]. 
Table 1. Main biomarker findings in familial AD and FTD

\begin{tabular}{|c|c|c|c|c|c|c|}
\hline \multirow[t]{2}{*}{ Gene } & \multicolumn{5}{|l|}{ CSF findings } & \multirow[t]{2}{*}{ References } \\
\hline & $\mathrm{A} \beta_{40}$ & $\mathrm{~A} \beta_{42}$ & t-tau & $\mathrm{p}$-tau & other proteins & \\
\hline \multirow[t]{2}{*}{$A P P$} & $=(2)$ & $\downarrow(9)$ & $\uparrow(8)$ & $\uparrow(7)$ & $\uparrow$ F2-isoprostanes (1), $\uparrow$ VILIP-1 (1) & $7-13,20,26$ \\
\hline & $=(1)$ & $\downarrow(1)$ & $=(1)$ & $=(1)$ & NA & 30 \\
\hline \multirow[t]{3}{*}{ PSEN1 } & $=(3) / \uparrow(1)$ & $\downarrow(22) /=(2)$ & $\uparrow(21)$ & $\uparrow(15)$ & $\uparrow$ albumin (1), $\uparrow$ F2-isoprostanes (1), $\uparrow$ & $8-23,26$ \\
\hline & & & & & VILIP-1 (1), 14-3-3 protein positivity (1) & \\
\hline & $=(1)$ & $\downarrow(3) / \uparrow(1)$ & $=(4)$ & $=(4)$ & NA & $27-30$ \\
\hline PSEN2 & $=(1)$ & $\downarrow(5)$ & $\uparrow(3)$ & $\uparrow(4)$ & $\uparrow$ VILIP-1 (1) & $8,11,13,24,25$ \\
\hline \multirow[t]{2}{*}{$M A P T$} & NA & $=(5)$ & $=(3) / \uparrow(4)$ & $=(3) / \uparrow(2)$ & NA & $26,33-35,58-60$ \\
\hline & NA & $=(3)$ & $=(3)$ & $=(3)$ & $\downarrow$ progranulin (1), $\uparrow$ pTDP-43 (1) & $38,39,61$ \\
\hline \multirow[t]{2}{*}{ GRN } & NA & $\downarrow(3)$ & $\uparrow(3)$ & $\uparrow(2)$ & NA & $26,36,37$ \\
\hline & NA & NA & NA & NA & $=$ progranulin $(1) / \downarrow$ progranulin $(1)$ & 62,63 \\
\hline \multirow[t]{2}{*}{ C9ORF72 } & NA & $\downarrow(2)$ & $\uparrow(2)$ & $\uparrow(2)$ & NA & 40,42 \\
\hline & $=(1)$ & $\downarrow(1) /=(2)$ & $=(3)$ & $=(3)$ & $\uparrow$ pTDP-43 (1) & $39,41,43$ \\
\hline
\end{tabular}

The table summarizes the CSF biomarker profiles found in mutation carriers of APP, PSEN1, PSEN2, MAPT, GRN or C90RF72. Numbers in parentheses denote the number of publications with the given biomarker finding. $\uparrow$ Levels increased in mutation carriers when compared with controls; $\downarrow$ levels decreased in mutation carriers when compared with controls; = levels unchanged in mutation carriers when compared with controls; NA = not assessed; pTDP-43 = phosphorylated TDP-43.

\section{Discussion}

The scientific community Alzforum (www.alzforum.org) has listed 223 reported mutations in PSEN1, 40 in APP and 37 in PSEN2, both pathogenic and non-pathogenic [44]. PSEN1 and PSEN2 are part of the $\gamma$-secretase complex, responsible for the generation of $A \beta$ from cleavage of APP to fragments of 39-42 amino acids, with $A \beta_{40}$ as the most common fragment. Mutations in PSEN1 or PSEN2 result in an increased production of $A \beta_{42}$ peptides, which are considered the more pathogenic and aggregation-prone form of $A \beta . A \beta_{42}$ peptides form toxic oligomers and aggregate into neuritic plaques, whereas soluble $A \beta_{42}$ harms synapses and causes inflammation, leading to synaptic injury and additional pathological events such as hyperphosphorylation of MAPT $[45,46]$. The hypothesis of an APP imbalance being involved in disease pathogenesis is supported by the observation that individuals born with Down's syndrome (trisomy 21) - thus having an extra copy of chromosome 21 , on which the APP gene is located - develop AD pathology with severe amyloid plaques $[47,48]$. Although there is a well-established triplet of CSF biomarkers used for AD diagnosis, there is a lack of biomarkers that reflect disease progression, early prediction and specificity against other dementias. The findings reviewed here show a similar biomarker profile amongst patients with inherited AD, with decreased CSF levels of $A \beta_{42}$ and increased p-tau and t-tau. This observation is not surprising, since these three biomarkers have specifically been developed for AD diagnosis and have shown to be specific for plaque and tangle pathology.

As for the familial FTD cases, the biomarker findings are more varied and do not reveal a clear picture of the underlying pathology (table 1). The differences in the CSF findings cannot be explained by the different mutations, since patients harbouring identical MAPT mutations are found to have opposing tau measurements in two unrelated studies [33, 35]. There are several possible explanations for the different observations. One reason might be age differences among study subjects; also differences in sample handling in the clinics and labora- 
Table 2. $A \beta$ isoform findings in familial $A D$

\begin{tabular}{|c|c|c|c|c|c|c|c|c|c|c|c|c|c|c|c|c|}
\hline \multirow[t]{2}{*}{ Gene } & \multicolumn{15}{|c|}{ Other $A \beta$ isoforms } & \multirow[t]{2}{*}{ Ref. } \\
\hline & $\mathrm{A} \beta_{13}$ & $\mathrm{~A} \beta_{14}$ & $\mathrm{~A} \beta_{15}$ & $\mathrm{~A} \beta_{16}$ & $\mathrm{~A} \beta_{17}$ & $A \beta_{18}$ & $\mathrm{~A} \beta_{19}$ & $\mathrm{~A} \beta_{20}$ & $\mathrm{~A} \beta_{30}$ & $\mathrm{~A} \beta_{33}$ & $\mathrm{~A} \beta_{34}$ & $\mathrm{~A} \beta_{37}$ & $\mathrm{~A} \beta_{38}$ & $A \beta_{39}$ & $\alpha$-sAPP & \\
\hline$A P P$ & NA & NA & NA & NA & NA & NA & NA & NA & NA & NA & NA & NA & NA & NA & $\downarrow$ & 64 \\
\hline PSEN1 & $=$ & $=$ & $=/ \uparrow$ & $=/ \uparrow$ & $=$ & $=$ & $=$ & $\uparrow$ & $=$ & $=$ & $=$ & $\downarrow$ & $\downarrow$ & $\downarrow$ & NA & 31,32 \\
\hline
\end{tabular}

$\uparrow$ Levels increased in mutation carriers when compared with controls; $\downarrow$ levels decreased in mutation carriers when compared with controls; = levels unchanged in mutation carriers when compared with controls; $\alpha$-sAPP = $\alpha$-soluble APP; NA=not assessed.

tories could affect outcome measurements, since a number of preanalytical factors have shown to influence the stability of CSF proteins [49]. Also, it was not possible to find a distinct biomarker profile which separates FTD cases with GRN mutations from sporadic cases based on $A \beta$, tau and p-tau levels [37]. This suggests that the underlying pathology of FTD is unrelated to the tau and $\mathrm{A} \beta$ pathology observed in $\mathrm{AD}$.

A specific and sensitive FTD marker has yet to be discovered. The literature reviewed here does not provide FTD markers as the findings are not consistent between the different studies, although some of the studies deal with individuals with the same mutation, especially among GRN mutation carriers. In sporadic FTD patients, TDP-43, progranulin and tau have been suggested as CSF biomarkers, but none of them has proven to be specific for differentiating FTD patients from AD or other dementia patients [4].

A weakness of the studies reviewed here is the very limited number of individuals included, with some of the studies being case studies. Biomarker findings in case reports on a single individual may not reflect overall or average changes found in patients with inherited AD or FTD, and this might also explain some of the opposing CSF findings in the reviewed studies shown in table 1 . The case reports have been included in this review due to the very limited number of large CSF biomarker studies on individuals with inherited AD or FTD, and we find it important to shed light on all CSF findings on the inherited AD/FTD dementias to date.

The strategy for developing AD biomarkers for clinical diagnostic use has been to assess whether the $\mathrm{A} \beta$ and tau pathology observed in postmortem $\mathrm{AD}$ brains as plaques and tangles is reflected in the CSF. Using a new approach, Ringman et al. [9] applied unbiased proteomics to study overall protein changes in presymptomatic and symptomatic individuals with familial AD carrying either PSEN1 or APP mutations. When compared, the proteomic profile of the mutation carriers showed 56 proteins significantly changed compared to non-carriers, including several markers of inflammation and synaptic loss. Forty-six proteins were found to be upregulated and 10 proteins were downregulated in familial AD mutation carriers compared to non-carriers. Interestingly, most of the participants in the study were presymptomatic, and changes in $A \beta$, tau and p-tau were detected 10 years or more before the age at onset. Further, several of the 56 proteins shown to be changed have been found to be altered in $\mathrm{AD}$ patients before, including APP, transferrin, $\alpha_{1} \beta$-glycoprotein, complement components, afamin precursor, spondin 1 and plasminogen. Additionally, Portelius and colleagues [31, 32] used immunoprecipitation mass spectrometry to investigate $A \beta$ isoforms in individuals with PSEN1 mutations and found that certain isoforms $\left(A \beta_{1-37}, A \beta_{1-38}\right.$ and $\left.A \beta_{1-39}\right)$ were decreased while $A \beta_{1-20}$ was increased in the CSF of both presymptomatic and symptomatic mutations carriers when compared to non-carriers (table 2). The data suggest that these proteins might be useful as biomarkers in presymptomatic and symptomatic individuals with APP and PSEN1 mutations. Further, the findings from Ringman et al. [9] indicate that other molecular mecha- 
Rostgaard et al.: Cerebrospinal Fluid Biomarkers in Familial Forms of Alzheimer's

Disease and Frontotemporal Dementia

nisms than $\mathrm{A} \beta$ and tau pathology are involved in development and progression of $\mathrm{AD}$ and point towards other possible treatment targets and strategies. The proteomic approach is interesting as a range of new potential biomarkers have been discovered which have not previously been under investigation. The method could be used when assessing changes in CSF proteins as the disease progresses; however, the results from both studies need to be replicated in a new and larger sample set.

Regarding FTD, only a few studies have investigated CSF with proteomics in sporadic FTD [50-52], and to our knowledge no studies on familial FTD have been published. Some of the proteins found to be changed comparing sporadic FTD patients and control individuals in the studies were albumin and transthyretin (upregulated) and truncated cystatin C, graninlike neuroendocrine precursor, retinol-binding protein and apolipoprotein E (downregulated). It would be interesting to assess if the same proteins are changed in individuals with familial FTD and whether proteomic changes can be detected decades before the onset of symptoms, as it seems to be the case in familial AD.

Yet another approach has been to assess the levels of inflammatory markers in the CSF using immunoassays. AD is characterized by upregulation of the brain's innate immune responses, resulting in inflammatory processes that orchestrate cytokine and cellular responses and culminate in neuronal injury and destruction [6]. In one of the reviewed studies, the levels of F2-isoprostanes, a marker of lipid peroxidation which is associated with inflammation, were increased in the CSF of both asymptomatic and symptomatic young APP or PSEN1 mutation carriers [20]. It remains unclear to what extend the inflammatory proteins are specifically linked to $\mathrm{AD}$ pathology, but these data suggest that inflammatory markers might have a potential role as early biomarkers in AD.

The ultimate goal would be to discover biomarkers reflecting early disease pathogenesis or predictors of disease development. Interestingly, Bateman et al. [11] and Fagan et al. [13] observed CSF biomarker abnormalities in autosomal dominant AD caused by mutations in PSEN1, PSEN2 or APP (the DIAN cohort) as early as 10-20 years before the estimated onset of symptoms, and mutation carriers developed a classic AD CSF profile the closer they were to the onset of symptoms. Further, Fagan et al. [13] found that VILIP-1, a marker of neuronal injury and cell death, was increased in mutation carriers up to 15 years before the estimated onset of symptoms. These studies and others $[10,28]$ demonstrate that disease pathogenesis in dementia most likely starts many years before the onset of dementia symptoms in individuals with autosomal dominant AD. It is therefore of great importance to discover specific biomarkers which can detect early disease pathogenesis and enable patients to begin medical evaluation and treatment in an attempt at potentially delaying the onset of symptoms.

\section{Conclusion}

In general, levels of $A \beta_{42}, t$-tau and $p$-tau are comparable between familial and sporadic $\mathrm{AD}$ patients and between familial and sporadic FTD patients, as shown in the biomarker findings reviewed here. These results as well as strong similarities in imaging markers and disease progression profiles suggest that some or most of the pathophysiology is shared between familial and sporadic forms of AD and FTD, respectively. The DIAN consortium has been established with the aim to study pathological changes in mutation carriers years before the onset of symptoms and to thereby determine how the AD process develops.

Individuals from the DIAN cohort are currently enrolled in a clinical drug trial with the anti-A $\beta$ antibodies gantenerumab (phase II trial) and solanezumab (phase III trial). The CSF markers which will be monitored in the solanezumab trial are $A \beta$ species, free $A \beta_{42}$ and $A \beta_{40}$ compared to total $A \beta_{42}$ and $A \beta_{40}$, as the antibody recognizes soluble $A \beta$. Gantenerumab is an 
Rostgaard et al.: Cerebrospinal Fluid Biomarkers in Familial Forms of Alzheimer's

Disease and Frontotemporal Dementia

antibody directed against aggregated $\mathrm{A} \beta$, and in this trial changes in $\mathrm{A} \beta$ peptide concentrations will be measured. Also changes in tau and $\mathrm{p}$-tau ${ }_{181}$, compared between subjects on the active drug and mutation carriers in the placebo group, will be measured in both trials [5355]. Despite clinical trials in sporadic AD individuals being cancelled prior to the end date as they failed to meet clinical endpoints, the two trials in the DIAN cohort are ongoing $[56,57]$. An initiative like DIAN is necessary for understanding the AD disease course and will contribute to clarifying a strategy for early diagnosis.

A similar consortium for studying familial FTDs caused by GRN, MAPT and C9ORF79 mutations, the GENFI consortium, has recently been established and is currently enrolling mutation carriers. It will be interesting to follow the work of GENFI and to see the impending results of their studies.

In view of the literature on biomarker research on familial AD and FTD presented here, further investigation of specific proteins and the overall protein content of the CSF is recommended, since this will provide a better understanding of the disease mechanisms causing neurodegeneration in AD and FTD and allow the discovery of new biomarkers for diagnosis.

\section{Acknowledgements}

This work was supported by funding from the 'Patient-specific stem cell-derived models for Alzheimer's disease' project of the Danish National Advanced Technology Foundation (N.R., G.W., J.E.N.) and from the Novo Nordisk Foundation (J.E.N.).

\section{Disclosure Statement}

The authors declare that there are no conflicts of interest regarding the publication of this review.

\section{References}

$>1$ World Health Organization; Alzheimer's Disease International: Dementia: a public health priority. Geneva, World Health Organization, 2012.

- Holtzman DM, Morris JC, Goate AM: Alzheimer's disease: the challenge of the second century. Sci Transl Med 2011;3:77sr1.

-3 Rossor MN, Fox NC, Mummery CJ, Schott JM, Warren JD: The diagnosis of young-onset dementia. Lancet Neurol 2010;9:793-806.

4 Hales CM, Hu WT: From frontotemporal lobar degeneration pathology to frontotemporal lobar degeneration biomarkers. Int Rev Psychiatry 2013;25:210-220.

5 Humpel C: Identifying and validating biomarkers for Alzheimer's disease. Trends Biotechnol 2011;29:26-32.

-6 Chintamaneni M, Bhaskar M: Biomarkers in Alzheimer's disease: a review. ISRN Pharmacol 2012;2012: 984786.

7 Ringman J, Gylys K, Medina L, Fox M: Biochemical, neuropathological, and neuroimaging characteristics of early-onset Alzheimer's disease due to a novel PSEN1 mutation. Neurosci Lett 2011;487:287-292.

-8 Wallon D, Rousseau S, Rovelet-Lecrux A, Quillard-Muraine M, Guyant-Maréchal L, Martinaud O, et al: The French series of autosomal dominant early onset Alzheimer's disease cases: mutation spectrum and cerebrospinal fluid biomarkers. J Alzheimers Dis 2012;30:847-856.

-9 Ringman JM, Schulman H, Becker C, Jones T, Bai Y, Immermann F, et al: Proteomic changes in cerebrospinal fluid of presymptomatic and affected persons carrying familial Alzheimer disease mutations. Arch Neurol 2012;69:96-104.

10 Ringman JM, Coppola G, Elashoff D, Rodriguez-Agudelo Y, Medina LD, Gylys K, et al: Cerebrospinal fluid biomarkers and proximity to diagnosis in preclinical familial Alzheimer's disease. Dement Geriatr Cogn Disord 2012;33:1-5.

11 Bateman RJ, Xiong C, Benzinger TLS, Fagan AM, Goate A, Fox NC, et al: Clinical and biomarker changes in dominantly inherited Alzheimer's disease. N Engl J Med 2012;367:795-804.

12 Schöll M, Wall A, Thordardottir S, Ferreira D, Bogdanovic N, Långström B, et al: Low PiB PET retention in presence of pathologic CSF biomarkers in Arctic APP mutation carriers. Neurology 2012;79:229-236. 
Rostgaard et al.: Cerebrospinal Fluid Biomarkers in Familial Forms of Alzheimer's

Disease and Frontotemporal Dementia

13 Fagan AM, Xiong C, Jasielec MS, Bateman RJ, Goate AM, Benzinger TLS, et al: Longitudinal change in CSF biomarkers in autosomal-dominant Alzheimer's disease. Sci Transl Med 2014;6:226ra30.

14 Luedecke D, Becktepe JS, Lehmbeck JT, Finckh U, Yamamoto R, Jahn H, et al: A novel presenilin 1 mutation (Ala275Val) as cause of early-onset familial Alzheimer disease. Neurosci Lett 2014;566:115-119.

-15 Testi S, Peluso S, Fabrizi GM, Antenora A, Russo CV, Pappatà S, et al: A novel PSEN1 mutation in a patient with sporadic early-onset Alzheimer's disease and prominent cerebellar ataxia. J Alzheimers Dis 2014;41:709-714.

16 Matsushita S, Arai H, Okamura N, Ohmori T, Takasugi K, Matsui T, et al: Clinical and biomarker investigation of a patient with a novel presenilin-1 mutation (A431V) in the mild cognitive impairment stage of Alzheimer's disease. Biol Psychiatry 2002;52:907-910.

-17 Fortea J, Lladó A, Bosch B, Antonell A, Oliva R, Molinuevo JL, et al: Cerebrospinal fluid biomarkers in Alzheimer's disease families with PSEN1 mutations. Neurodegener Dis 2011;8:202-207.

18 Aoki M, Abe K, Oda N, Ikeda M, Tsuda T, et al: A presenilin-1 mutation in a Japanese family with Alzheimer's disease and distinctive abnormalities on cranial MRI. Neurology 1997;48:1118-1120.

19 Balasa M, Vidal-Piñeiro D, Lladó A, Antonell A, Bosch B, Castellanos F, et al: PSEN1 mutation carriers present lower cerebrospinal fluid amyloid- $\beta_{42}$ levels than sporadic early-onset Alzheimer's disease patients but no differences in neuronal injury biomarkers. J Alzheimers Dis 2012;30:605-616.

20 Ringman JM, Younkin SG, Pratico D, Seltzer W, Cole GM, Geschwind DH, et al: Biochemical markers in persons with preclinical familial Alzheimer disease. Neurology 2008;71:85-92.

-21 Antonell A, Balasa M, Oliva R, Lladó A, Bosch B, Fabregat N, et al: A novel PSEN1 gene mutation (L235R) associated with familial early-onset Alzheimer's disease. Neurosci Lett 2011;496:40-42.

22 de Bot ST, Kremer HPH, Dooijes D, Verbeek MM: CSF studies facilitate DNA diagnosis in familial Alzheimer's disease due to a presenilin-1 mutation. J Alzheimers Dis 2009;17:53-57.

-23 Tiedt HO, Lueschow A, Winter P, Müller U: Previously not recognized deletion in presenilin-1 (p.Leu174del.) in a patient with early-onset familial Alzheimer's disease. Neurosci Lett 2013;544:115-118.

24 Binetti G, Signorini S, Squitti R, Alberici A, Benussi L, Cassetta E, et al: Atypical dementia associated with a novel presenilin-2 mutation. Ann Neurol 2003;54:832-836.

-25 Nikisch G, Hertel A, Kiessling B, Wagner T, Krasz D, Hofmann E, Wiedemann G: Three-year follow-up of a patient with early-onset Alzheimer's disease with presenilin-2 N141I mutation - case report and review of the literature. Eur J Med Res 2008;13:579-584.

-26 Cohn-Hokke PE, Wong TH, Rizzu P, Breedveld G, van der Flier WM, Scheltens P, et al: Mutation frequency of PRKAR1B and the major familial dementia genes in a Dutch early onset dementia cohort. J Neurol 2014;261: 2085-2092.

-27 Uttner I, Kirchheiner J, Tumani H, Mottaghy FM, Lebedeva E, Ozer E, et al: A novel presenilin1 mutation (Q223R) associated with early onset Alzheimer's disease, dysarthria and spastic paraparesis and decreased Abeta levels in CSF. Eur J Neurol 2010;17:631-633.

28 Reiman EM, Quiroz YT, Fleisher AS, Chen K, Velez-Pardo C, Jimenez-Del-Rio M, et al: Brain imaging and fluid biomarker analysis in young adults at genetic risk for autosomal dominant Alzheimer's disease in the presenilin 1 E280A kindred: a case-control study. Lancet Neurol 2012;11:1048-1056.

29 Moonis M, Swearer J, et al: Familial Alzheimer disease: decreases in CSF A $\beta_{42}$ levels precede cognitive decline. Neurology 2005;65:323-325.

-30 Almkvist 0, Axelman K, et al: Clinical findings in nondemented mutation carriers predisposed to Alzheimer's disease: a model of mild cognitive impairment. Acta Neurol Scand Suppl 2003;179:77-82.

-31 Portelius E, Andreasson U, Ringman JM, Buerger K, Daborg J, Buchhave P, et al: Distinct cerebrospinal fluid amyloid beta peptide signatures in sporadic and PSEN1 A431E-associated familial Alzheimer's disease. Mol Neurodegener 2010;5:2.

-32 Portelius E, Fortea J, Molinuevo JL, Gustavsson MK, Andreasson U, Sanchez-Valle R: The amyloid- $\beta$ isoform pattern in cerebrospinal fluid in familial PSEN1 M139T- and L286P-associated Alzheimer's disease. Mol Med Rep 2012;5:1111-1115.

-33 Lindquist SG, Holm IE, Schwartz M, Law I, Stokholm J, Batbayli M, et al: Alzheimer disease-like clinical phenotype in a family with FTDP-17 caused by a MAPT R406W mutation. Eur J Neurol 2008;15:377-385.

34 Rosso SM, van Herpen E, Pijnenburg YA, Schoonenboom NS, Scheltens P, Heutink P, et al: Total tau and phosphorylated tau 181 levels in the cerebrospinal fluid of patients with frontotemporal dementia due to P301L and G272V tau mutations. Arch Neurol 2003;60:1209-1213.

-35 Tolboom N, Koedam E, et al: Dementia mimicking Alzheimer's disease owing to a tau mutation: CSF and PET findings. Alzheimer Dis Assoc Disord 2010;24:303-307.

-36 Brouwers N, Nuytemans K, et al: Alzheimer and Parkinson diagnoses in progranulin null mutation carriers in an extended founder family. Arch Neurol 2007;64:1436-1446.

-37 Carecchio M, Fenoglio C, Cortini F, Comi C, Benussi L, Ghidoni R, et al: Cerebrospinal fluid biomarkers in progranulin mutations carriers. J Alzheimers Dis 2011;27:781-790.

-38 Pietroboni A, Fumagalli G, et al: Phenotypic heterogeneity of the GRN Asp22fs mutation in a large Italian kindred. J Alzheimers Dis 2011;24:253-259.

-39 Suárez-Calvet M, Dols-Icardo O, Lladó A, Sánchez-Valle R, Hernández I, Amer G, et al: Plasma phosphorylated TDP-43 levels are elevated in patients with frontotemporal dementia carrying a C9orf72 repeat expansion or a GRN mutation. J Neurol Neurosurg Psychiatry 2014;85:684-691. 
Rostgaard et al.: Cerebrospinal Fluid Biomarkers in Familial Forms of Alzheimer's

Disease and Frontotemporal Dementia

40 Wallon D, Rovelet-Lecrux A, Deramecourt V, Pariente J, Auriacombe S, Le Ber I, et al: Definite behavioral variant of frontotemporal dementia with C9ORF72 expansions despite positive Alzheimer's disease cerebrospinal fluid biomarkers. J Alzheimers Dis 2012;32:19-22.

-41 Suhonen NM, Kaivorinne AL, Moilanen V, Bode M, Takalo R, Hänninen T, et al: Slowly progressive frontotemporal lobar degeneration caused by the C9ORF72 repeat expansion: a 20-year follow-up study. Neurocase 2015;21:85-89.

-42 Saint-Aubert L, Sagot C, Wallon D, Hannequin D, Payoux P, Nemmi F, et al: A case of logopenic primary progressive aphasia with C9ORF72 expansion and cortical florbetapir binding. J Alzheimers Dis 2014;42:413420.

43 Abbate C, Arosio B, Galimberti D, Nicolini P, Chiara LR, Rossi PD, et al: Phenotypic variability associated with the C9ORF72 hexanucleotide repeat expansion: a sporadic case of frontotemporal lobar degeneration with prodromal hyposmia and predominant semantic deficits. J Alzheimers Dis 2014;40:849-855.

44 Alzforum: Mutation database. http://www.alzforum.org/mutations.

45 Shen J, Kelleher RJ 3rd: The presenilin hypothesis of Alzheimer's disease: evidence for a loss-of-function pathogenic mechanism. Proc Natl Acad Sci USA 2007;104:403-409.

46 Gendron TF, Petrucelli L: The role of tau in neurodegeneration. Mol Neurodegener 2009;4:13.

$\checkmark 47$ Haass C, Selkoe DJ: Soluble protein oligomers in neurodegeneration: lessons from the Alzheimer's amyloid beta-peptide. Nat Rev Mol Cell Biol 2007;8:101-112.

48 Jakob-Roetne R, Jacobsen H: Alzheimer's disease: from pathology to therapeutic approaches. Angew Chem Int Ed Engl 2009;48:3030-3059.

49 Simonsen AH, Bahl JMC, Danborg PB, Lindstrom V, Larsen SO, Grubb A, et al: Pre-analytical factors influencing the stability of cerebrospinal fluid proteins. J Neurosci Methods 2013;215:234-240.

50 Rüetschi U, Zetterberg H, Podust VN, Gottfries J, Li S, Hviid Simonsen A, et al: Identification of CSF biomarkers for frontotemporal dementia using SELDI-TOF. Exp Neurol 2005;196:273-281.

-51 Davidsson P, Sjögren M, Andreasen N, Lindbjer M, Nilsson CL, Westman-Brinkmalm A, et al: Studies of the pathophysiological mechanisms in frontotemporal dementia by proteome analysis of CSF proteins. Mol Brain Res 2002;109:128-133.

52 Simonsen A, McGuire J, Podust VN, Hagnelius N-O, Nilsson TK, Kapaki E, et al: A novel panel of cerebrospinal fluid biomarkers for the differential diagnosis of Alzheimer's disease versus normal aging and frontotemporal dementia. Dement Geriatr Cogn Disord 2007;24:434-440.

53 ClinicalTrials.gov: Dominantly Inherited Alzheimer Network trial: an opportunity to prevent dementia. A study of potential disease modifying treatments in individuals at risk for or with a type of early onset Alzheimer's disease caused by a genetic mutation (DIAN-TU). 2012. https://www.clinicaltrials.gov/ct2/ show/NCT01760005?term=NCT01760005\&rank=1 (accessed February 19, 2015).

54 ClinicalTrials.gov: Dominantly Inherited Alzheimer Network (DIAN). 2009. https://www.clinicaltrials.gov/ ct2/show/NCT00869817?term=NCT00869817\&rank=1 (accessed February 19, 2015).

55 Moulder KL, Snider BJ, Mills SL, Buckles VD, Santacruz AM, Bateman RJ, et al: Dominantly Inherited Alzheimer Network: facilitating research and clinical trials. Alzheimers Res Ther 2013;5:48.

56 Doody RS, Thomas RG, Farlow M, Iwatsubo T, Vellas B, Joffe S, et al: Phase 3 trials of solanezumab for mild-tomoderate Alzheimer's disease. N Engl J Med 2014;370:311-321.

57 Roche: Media release: Roche provides update on gantenerumab development programme. 2014. http://www. roche.com/media/store/releases/med-cor-2014-12-19b.htm.

-58 Iovino M, Pfisterer U, Holton JL, Lashley T, Swingler RJ, Calo L, et al: The novel MAPT mutation K298E: mechanisms of mutant tau toxicity, brain pathology and tau expression in induced fibroblast-derived neurons. Acta Neuropathol 2014;127:283-295.

59 Ishida C, Kobayashi K, Kitamura T, Ujike H, Iwasa K, Yamada M: Frontotemporal dementia with parkinsonism linked to chromosome 17 with the MAPT R406W mutation presenting with a broad distribution of abundant senile plaques. Neuropathology 2015;35:75-82.

60 Popović M, Fabjan A, Mraz J, Magdič J, Glavač D, Zupan A, et al: Tau protein mutation P364S in two sisters: clinical course and neuropathology with emphasis on new, composite neuronal tau inclusions. Acta Neuropathol 2014;128:155-157.

61 Calvi A, Cioffi SMG, Caffarra P, Fenoglio C, Serpente M, Pietroboni AM, et al: The novel GRN g.1159_1160delTG mutation is associated with behavioral variant frontotemporal dementia. J Alzheimers Dis 2015;44:277-282.

62 Ghidoni R, Benussi L, Glionna M, Franzoni M, Binetti G: Low plasma progranulin levels predict progranulin mutations in frontotemporal lobar degeneration. Neurology 2008;71:1235-1239.

63 Philips T, De Muynck L, Thu HN, Weynants B, Vanacker P, Dhondt J, et al: Microglial upregulation of progranulin as a marker of motor neuron degeneration. J Neuropathol Exp Neurol 2010;69:1191-1200.

64 Lannfelt L, Basun H, Wahlund L, et al: Decreased $\alpha$-secretase-cleaved amyloid precursor protein as a diagnostic marker for Alzheimer's disease. Nat Med 1995;1:829-832. 